\title{
Role of circulating miR 194-5p, miR 106b, and miR 146a as potential biomarkers for epilepsy: a case-control study
}

\author{
Mervat Moustafa', Noha T. Abokrysha', Nermeen A. Eldesoukey², Dalia Gamil Amin², Nesma Mounir ${ }^{1}$ and \\ Dalia M. Labib ${ }^{1 *}$
}

\begin{abstract}
Background: Epilepsy is a chronic neurological disease. A suitable biomarker for epilepsy diagnosis remains lacking. MicroRNAs (miRNAs) were pronounced as promising biomarkers for epileptogenesis.

Objectives: To analyze the expression levels of miR 194-5p, miR 106b, and miR 146a in Egyptian epileptic patients compared to control subjects and to detect their correlation to clinical characteristics.

Subjects and methods: We evaluated the expression levels of miR 106b, miR 146a, and miR 194-5p using real-time quantitative polymerase chain reaction (qRT-PCR) in 50 subjects: 15 patients with idiopathic generalized epilepsy, 15 patients with focal epilepsy (3 idiopathic and 12 cryptogenic), and 20 healthy controls.
\end{abstract}

Results: miR 106b and miR 194-5p were upregulated in the generalized epilepsy group compared to control; miR 194-5p was significantly downregulated in the focal epilepsy group compared to the generalized epilepsy group and control ( $p^{<}$0.05). miR 194-5p was negatively correlated to disease duration in patients with focal epilepsy; the three microRNAs were positively correlated to each other ( $\left.p^{<} 0.05\right)$.

Conclusion: Serum miR 194-5P and miR 106b can be used as potential non-invasive biomarkers in the evaluation of idiopathic generalized epilepsy.

Keywords: Epilepsy, miR 194-5p, miR 146a, miR 106b, Biomarker

\section{Introduction}

Epilepsy is the fourth common neurological disease represented by repeated unprovoked seizures, secondary to episodes of temporary brain dysfunction secondary to abnormal electrical activity. Epilepsy represents a high social burden all over the world, with a prevalence of 10.3 per 1000 in developing countries [1].

The different and complex pathophysiological mechanism is implicated in each epilepsy syndrome, even in symptomatic epilepsies [2]. Biomarkers are needed for accurate diagnosis and treatment, to detect the process

\footnotetext{
* Correspondence: dr.dalia.neuro@gmail.com

${ }^{1}$ Neurology Department, Faculty of Medicine, Cairo University, Cairo, Egypt

Full list of author information is available at the end of the article
}

of epileptogenesis and disease progression before the first seizure occur, a reliable biomarker can detect the epileptogenic process after the first seizure to help start treatment without waiting for the subsequent seizure, a specific biomarker can detect the epileptogenic zone which helps for accurate presurgical evaluation, and also a biomarker is important to detect the response of therapeutic intervention and early detection of patients with drug-resistant epilepsy [3].

Diagnosis of epilepsy depends on a detailed medical history, clinical examination, electroencephalogram, and neuroimaging. Usually, these methods are not sufficient and also expensive [4]. Noninvasive, rapid, and more economical biomarkers are still in need of a more accurate diagnosis of epilepsy [5].

\section{Springer Open}

(c) The Author(s). 2020 Open Access This article is licensed under a Creative Commons Attribution 4.0 International License, which permits use, sharing, adaptation, distribution and reproduction in any medium or format, as long as you give appropriate credit to the original author(s) and the source, provide a link to the Creative Commons licence, and indicate if changes were made. The images or other third party material in this article are included in the article's Creative Commons licence, unless indicated otherwise in a credit line to the material. If material is not included in the article's Creative Commons licence and your intended use is not permitted by statutory regulation or exceeds the permitted use, you will need to obtain permission directly from the copyright holder. To view a copy of this licence, visit http://creativecommons.org/licenses/by/4.0/. 
MicroRNAs (miRNAs) are classes of small non-coding RNAs elaborated in different physiological and developmental functions; their dysregulation was linked to many diseases and have been suggested as new biomarkers for different diseases [6-9].

In the recent decade, some studies tried to investigate the role of microRNAs in the different mechanisms of the epileptogenesis process as in inflammation, synaptic remodeling, neuronal differentiation, and proliferation. More than 100 dysregulated miRNAs in the serum of epileptic patients had been identified and were related to epileptogensis $[10,11]$. These findings introduced microRNAs as potential diagnostic biomarkers for epilepsy and furtherly could be used as a potential therapeutic target [5].

The miR 146a and miR 194-5p regulate inflammation or apoptosis [12-14]. They are upregulated in several diseases associated with activation of the inflammatory responses. The miR 146a is transcriptionally induced by nuclear factor kappa-B (NF-B) in response to activation of innate immune signaling and has an important role in immune function regulation [15]. Also, it has been involved in the modulation of innate immunity through regulation of Toll-like receptor (TLR) signaling and cytokine responses [15]. The miR 194-5p was studied in gene regulation of cancer cells and was involved in cell differentiation, cell proliferation, and apoptosis by targeting different genes $[15,16]$. The role of miR 106b in epileptogenesis is still under research.

Accordingly, we aimed to analyze the expression levels of miR 106b, miR 146a, and miR 194-5p in Egyptian epileptic patients and to compare them to control subjects and to detect their correlation to clinical characteristics.

\section{Subjects and methods}

Our case-control study was performed on 50 adult Egyptian subjects. One month after the last seizure [17], serum samples were obtained from 30 epileptic patients (7 males and 23 females) with a mean age of $31.37 \pm$ 9.31 years. Patients were recruited from Cairo University Epilepsy Unit (CUEU), Kasr Alainy Hospitals, Cairo University, from June 2017 to June 2018. The epileptic patients were further divided into 2 subgroups according to guidelines of the International League Against Epilepsy (ILAE), based on electroclinical findings (seizure semiology and EEG) and etiology [18].

Group (I) included 15 patients with generalized epilepsy ( 3 males and 12 females) with a mean age of $27.47 \pm 7.25$ years.

Group (II) included 15 patients with focal epilepsy (4 males and 11 females) with a mean age of $35.27 \pm$ 9.72 years.

Epileptic patients with a history of systemic or central nervous system (CNS) infection within the last 2 weeks before sample collection, immune deficiency disorder [19], diabetes mellitus [20], cerebrovascular diseases, and autoimmune diseases were excluded [21].

The healthy sera were collected from 20 age-matched healthy individuals who volunteered to serve as a control group ( 9 males and 11 females) with a mean age of $33 \pm$ 9.31 years.

This was a conjoint work between the Neurology Department and Clinical Pathology Department of Kasr Alainy Medical School at Cairo University.

Informed consent was signed by all subjects and our study was approved by the ethical committee of the Faculty of Medicine, Cairo University (according to the WMA Declaration of Helsinki).

All patients were subjected to careful medical history and general and neurological examination with special emphasizing on age of onset, type of epilepsy, seizure semiology, duration of epilepsy, family history of epilepsy, history of any comorbidities, antiepileptic treatment (types, doses, compliance, side effects, frequency with compliance), and frequency of seizures during the last 12 months; the patient was considered having frequent fits if he had more than one fit in a month, and the patient was considered having infrequent fits if he had one fit or less in a month, disease control; the patient was considered controlled if he was fit-free $\geq 12$ months.

Five-milliliter whole blood was collected into a plain vacutainer tube from each subject and processed in the Clinical Pathology Department, Cairo University. Serum isolation was done by centrifugation at $1600 \mathrm{rpm}$ for 15 min at room temperature. The supernatant was shifted to Eppendorf tubes. The samples were recentrifuged at $14,000 \mathrm{rpm}$ for $10 \mathrm{~min}$ to precipitate cell debris and the supernatants were stored at $-80{ }^{\circ} \mathrm{C}$ until the extraction of ribonucleic acid (RNA).

These samples were used for detection of the expression level of mature miR 146a, miR 106b, and miR 194$5 p$, with cel-miR-39, which was used as a reference gene for normalization [22, 23]. RNA for miRNA expression was isolated from serum using the miRNeasy Mini kit (Qiagen, catalog no.217004). The concentration and purity of RNA were determined by measuring the absorbance at 260 and $280 \mathrm{~nm}$ using NanoQuant (infinite M200) Spectrophotometer (Tecan, Grodig, Australia). We used the manufacturer's protocol for total RNA extraction including small RNA.

Reverse transcription (RT) was implemented using the miScript II RT kit Quantitative Reverse transcription real-time polymerase chain reaction (q-RT-PCR) in $20 \mu \mathrm{L}$ reaction volume including $12 \mu \mathrm{L}$ templates RNA. Complementary deoxyribonucleic acid (cDNA) dilution and reaction mix preparation using miScript SYBR Green I PCR kit protocol (total volume $25 \mu \mathrm{L}$ including 
12.5 $\mu \mathrm{L}$ SYBR Green I PCR master mix, $2.5 \mu \mathrm{L}$ miScript universal primer, $2.5 \mu \mathrm{L}$ miScript primer assay, $5 \mu \mathrm{L}$ template cDNA). All reactions were run using 7500 realtime PCR system (Applied Biosystems, USA) using the following cycling conditions: $95^{\circ} \mathrm{C}$ for $15 \mathrm{~min}, 40$ cycles at $94{ }^{\circ} \mathrm{C}$ for $15 \mathrm{~s}, 55^{\circ} \mathrm{C}$ for $30 \mathrm{~s}$, and $70^{\circ} \mathrm{C}$ for $30 \mathrm{~s}$. All used primer assays in miScript SYBR Green I PCR kit were from QIAGEN: miR 146a (Cat. no. \#MS00003535), miR 106b (Cat. no. \#MS00003402), miR 194-5p (Cat. no. \#MS00006727), and the internal control gene cel-miR 39 (Cat. no. \#MS00019789). Relative expression of studied miRNA was calculated using the equation $2^{-\varangle x c t}$.

\section{Statistical analysis}

Data were described in terms of mean \pm standard deviation $( \pm \mathrm{SD})$, median and range for quantitative variables, and frequencies (number of patients) and relative frequency (percentage) for categorical data. KruskalWallis and Mann-Whitney tests were used to compare quantitative variables. The one-way analysis of variance (ANOVA) with post hoc test was used to compare more than 2 groups. For comparing categorical data, chi-square (c2) test was performed and when the expected frequency was less than 5 , the exact test was used instead. Odds ratio (OR) with 95\% confidence intervals was calculated [24]. The Spearman correlation coefficient was used to correlate quantitative variables. The receiver operating characteristic (ROC) curve was constructed with the area under curve analysis performed to detect the best cutoff value of the detection of the miR 106 and the miR 194 as a protective value of upregulation. $p$ values $<0.05$ were considered as statistically significant. Statistical calculations were performed using computer programs SPSS (Statistical Package for the Social Science; SPSS Inc., Chicago, IL, USA) version 25 for Microsoft Windows.

\section{Results}

Demographic data and clinical characteristics of the patients in groups I and II have been presented in Table 1.

As regard epileptic syndromes, group I included 15 patients with idiopathic generalized epilepsy, 8 patients with juvenile myoclonic epilepsy, 5 patients with epilepsy with generalized tonic-clonic seizures on awakening, and 2 patients with childhood absence epilepsy while group II included 12 patients with cryptogenic focal epilepsy (9 temporal lobe epilepsy and 3 frontal lobe epilepsy) and 3 patients with autosomal dominant nocturnal frontal lobe epilepsy (ADNFLE).

As regard seizures types, in group I, there were 11 patients (73.3\%) with myoclonus, 6 patients (40\%) with tonic, and 15 patients (100\%) with tonic-clonic seizures, while in group II, there were 9 patients (60\%) with complex partial seizures, 7 patients $(46.7 \%)$ with simple partial seizures, and 12 of patients $(80 \%)$ with focal fits with secondary generalization.

Regarding interictal EEG findings, 15 patients of group I had generalized epileptiform discharge, while in group II, 13 patients had focal discharges (3 frontotemporal, 2 frontocenral, 4 temporal, and 1 frontal), and 2 patients had normal EEG. All epileptic patients had normal MRI brain.

Two patients (13.3\%) in group I and 3 patients (20\%) in group II were controlled (fit-free $>1$ year), while 13 patients $(86.7 \%)$ in group I and 12 patients (80\%) in group II were not controlled.

Five patients (33.3\%) in group I and 6 patients (40\%) in group II were on monotherapy while 10 patients $(66.7 \%)$ in group I and 9 patients $(60 \%)$ in group II were on polytherapy.

As regard antiepileptic drugs (AEDs), 13 patients are on valproate with range of dose $(600-1800 \mathrm{mg} /$ day $), 4$ patients on lamotrigine $(100-200 \mathrm{mg} / \mathrm{d}), 11$ patients on levetiracetam (1000-4000 mg/day), 16 patients on carbamazepine $(100-800 \mathrm{mg} /$ day), 4 patients on phenytoin (200-300 mg/day). In group I, the higher percentage of patients were receiving valproate $(66.7 \%)$, followed by levetiracetam, lamotrigine, and topiramate as added on therapy, while in group II, the highest percentage of patients were receiving carbamazepine (93.4\%), followed by valproate, lamotrigine, levetiracetam, and phenytoin.

The mean values of microRNA-194-5p, 106b, and $146 \mathrm{a}$ of the epileptic patients were nonsignificant

Table 1 clinical and demographic results of the patients in groups I and ||

\begin{tabular}{|c|c|c|c|}
\hline & Group I & Group II & $\boldsymbol{p}$ value \\
\hline & Mean \pm SD & Mean \pm SD & \\
\hline Age (yrs.) & $27.47 \pm 7.25$ & $35.27 \pm 9.72$ & $0.015^{*}$ \\
\hline Age of onset (yrs.) & $16.53 \pm 5.67$ & $17.47 \pm 9.20$ & 0.870 \\
\hline BMI & $23.96 \pm 2.13$ & $23.36 \pm 2.31$ & 0.412 \\
\hline Disease duration (yrs.) & $10.93 \pm 7.07$ & $17.80 \pm 11.63$ & 0.089 \\
\hline Seizure frequency & $28.64 \pm 3.21$ & $13.19 \pm 25.49$ & 0.724 \\
\hline Positive family history of epilepsy, $N(\%)$ & $4(26.7 \%)$ & $4(26.7 \%)$ & 0.356 \\
\hline
\end{tabular}

$B M I$ body mass index, $N$ number, $S D$ standard deviation, yrs. years

*Significant $p<0.05$ 
dysregulated compared to the mean values of the control subjects $\left(p^{>} 0.05\right)$.

The comparison between groups I and II and control subjects as regards the differential expression of miRNAs was illustrated in Table 2 and Fig. 1.

In group I, the ROC curve revealed that the miR 194$5 \mathrm{p}$ and miR 106b values of 1.497 and 2.46 (respectively) can be used as cutoff values to diagnose epilepsy with significant $p$ values (0.039 and 0.021 , respectively). The sensitivity of the cutoff value of miR 194-5p was $73.3 \%$ with $55.6 \%$ specificity and the area under the curve (AUC) was 0.711 with a confidence interval (53-88\%), while the sensitivity of the cutoff value of miR $106 \mathrm{~b}$ was $60 \%$ with $66.7 \%$ specificity and the area under the curve (AUC) was (0.73) with a confidence interval (CI) 56$90 \%$.

By nominal comparison of data by classifying the values into upregulated and downregulated values, there was no statistically significant difference between epileptic patients and control or between subgroups and control, apart from miR 194-5p in group 2. The expression of miR 194-5p was significantly downregulated in group $2(12 / 15$ patients downregulated $80 \%)$ in comparison to group I and control subjects $(p=0.03)$. The odds ratio of miR 194-5p was 0.2 with a confidence interval (CI 0.04-0.95), which indicates the protective value of its upregulation.

On comparing the expression of the 3 miRNAs in controlled to uncontrolled patients, the mean expression of miR 106b, miR 146a, and miR 194-5p in group I were upregulated in uncontrolled patients $(5.31 \pm 7.53,1.30 \pm$ 0.82 , and $5.41 \pm 9.22$, respectively) compared to controlled patients $(1.17 \pm 0.45,0.96 \pm 0.11$, and $1.38 \pm$ 0.82 , respectively).

In group II, the mean expression of miR 106b, miR $146 \mathrm{a}$, and $\mathrm{miR} 194-5 \mathrm{p}$ were upregulated in controlled patients $(1.14 \pm 0.93,1.28 \pm 1.44$, and $1.01 \pm 0.81$, respectively) compared to uncontrolled patients $(0.67 \pm$ $0.71,1.26 \pm 0.9$, and $0.71 \pm 0.82$, respectively). However, no significant difference was found in the 3 microRNA expression of controlled to uncontrolled patients in either group I or group II $\left(p^{>} 0.05\right)$.

Table 2 Comparison between groups I and II and control subjects as regards the expression of miR 106b, miR 146a, and miR 194-5p

\begin{tabular}{lllll}
\hline & Group I & Group I & Control & $\boldsymbol{p}$ value \\
& Mean \pm SD & Mean \pm SD & Mean \pm SD & \\
\hline miR 106b & $2.99 \pm 2.09$ & $0.77 \pm 0.75$ & $1.61 \pm 1.30$ & $0.02^{*}$ \\
miR 146a & $1.25 \pm 0.77$ & $1.27 \pm 0.97$ & $0.97 \pm 0.41$ & 0.847 \\
miR 194-5p & $2.68 \pm 1.84$ & $0.78 \pm 0.79$ & $1.55 \pm 1.27$ & $0.04^{*}$ \\
\hline
\end{tabular}

miR microRNA, SD standard deviation

*Significant $p<0.05$
On comparing miRNA expression in patients receiving monotherapy and those receiving polytherapy, in group I, miR 106b and miR 194-5p mean expressions were upregulated in patients receiving monotherapy (7.45 \pm 12.34 and $8.56 \pm 15.07$, respectively) compared to those receiving polytherapy $(3.41 \pm 2.26$ and $3.03 \pm 2.07$, respectively), while $\mathrm{miR}$ 146a mean expression was upregulated in polytherapy patients $(1.34 \pm 0.74)$ compared to monotherapy patients $(1.07 \pm 0.87)$. In group II, we found that the mean expression of miR 106b, miR 146a, and $\mathrm{miR} 194-5 \mathrm{p}$ were higher in polytherapy patients $(0.90 \pm 0.89,1.52 \pm 1.13$, and $0.94 \pm 0.95$, respectively $)$ compared to monotherapy patients $(0.54 \pm 0.35,0.82 \pm$ 0.32 , and $0.49 \pm 0.28$, respectively). However, all the values were not reaching the statistical significant difference between the subgroups $\left(p^{>} 0.05\right)$.

miR 194-5p was negatively correlated to disease duration in group II $(r=-0.527, p=0.044)$; otherwise, no significant correlation was found in either group between these 3 miRNAs and patients' age, gender, BMI, the age at onset of epilepsy, duration of epilepsy, or seizure frequency $\left(p^{>} 0.05\right)$.

The dysregulated miR 106b, 146a, and 194-5p were positively correlated to each other in both groups I and II (Fig. 2)

\section{Discussion}

Epilepsy is the fourth most common neurological disease. Idiopathic epilepsies are groups of electroclinical syndromes with a presumed genetic etiology with complex inheritance patterns [25]. The epigenetic role in controlling the different genes involved in the pathogenesis of idiopathic epilepsies is still under research. MicroRNAs are small RNAs that have an epigenetic role by posttranscriptional inhibition of specific target genes [10]. The measurement of microRNA levels and the prediction of the target messenger RNA (mRNA) are required for the understanding of their role in epileptogenesis process.

Our study aimed to analyze and to deeply investigate the levels of miR 194-5p, 106b, and 146a in idiopathic epilepsy to present them as potential diagnostic biomarkers for epilepsy.

Our results revealed dysregulation of the miR 194-5p, miR 106b, and miR 146a of epileptic patients compared to the control subjects with no statistically significant difference between them. On studying the microRNA among the 2 groups, we found significant upregulation of miR 106b in patients with idiopathic generalized epilepsy (group I) and its downregulation in patients with idiopathic focal epilepsy (group II) in comparison to control subjects. Our results go in concordance with the results of many previous studies [23, 26, 27]. 


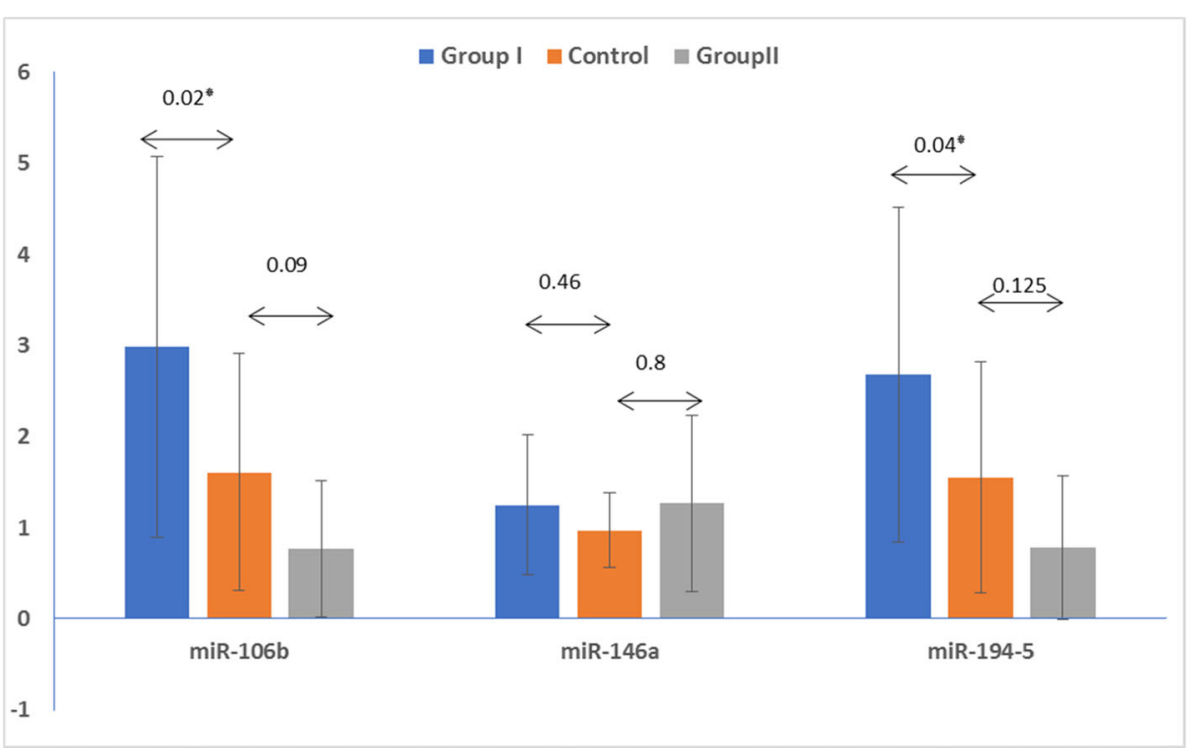

Fig. 1 Comparison between groups I and II and control as regards the expression of miR 106b, miR 146a, and miR 194-5p. *Significant $p$ value

miR 106b was found to be linked to inflammation, apoptosis in different CNS diseases. It facilitates the neuroinflammation process by targeting Transforming growth factor-beta (TGF $\beta$ ) [28]. TGF $\beta$ was found to be linked to neuroinflammation in epilepsy through activation of transforming growth factor beta-activated kinase 1 (TAK1), a key component of inflammation signaling in epilepsy [29]. TGF $\beta$ displays neuroprotective functions through anti-apoptotic, anti-excitotoxic actions, and the promotion of neurodegeneration [30]. Targeting TGF $\beta$ and its posttranscriptional inhibition by miR 106b suggest a proepileptogenic role of miR $106 \mathrm{~b}$.

Also, miR 106b had been linked to epilepsy-associated neurodegeneration and cognitive dysfunction [31]. It has a role in the apoptosis process as a pro-apoptotic factor by activating the expression of caspase- 3 and caspase- 9 in brain cells [32]. It was linked to caspase-6 as a predicted target gene in epileptic patients [23]. Caspase-6 was found to be related to programmed cell death in the epileptogenic hippocampus and remains elevated long after the occurrence of acute cell death during the epileptogenesis [33].

Another important mechanism, miR 106b is linked to the epilepsy-associated proliferation and neurogenesis [10]. Brett and coworkers noticed the association between the upregulation of $\mathrm{miR} 106 \mathrm{~b}$ and increased neural stem proliferating cells and neuronal differentiation [34].

We attribute the differential pattern of miR 106b in groups I and II to the different epigenetic regulatory roles of microRNAs in idiopathic generalized epilepsies that differ from that in idiopathic focal epilepsies.

In agreement with previous studies [23, 27]; we found non-significant upregulation of the miR 146a in the two epileptic groups I and II compared to control subjects.

Previously, the role of miR 146a in epileptogenesis was studied and it was related to the regulation of inflammation [16, 35] and the immune system [36, 37]. Aronica and colleagues identified an anti-epileptogenic role of miR 146a through negative regulation of inflammation via suppressing interleukin-6 (IL-6) and cyclooxygenase-2 (COX-2) signaling mediated by astrocytes [12]. Omran and colleagues noticed that the process of inflammation is triggered by the elevation of interleukin-1 $\beta$ (IL-1) that upregulated the miR 146a which in turn downregulated the inflammatory response [13]. miR 146a has a potential endogenous regulatory role on Toll-like receptor (TLR) and cytokine receptor signaling; it regulates IL-1 receptor-associated kinases (IRAK1, 2) and TNF receptor-associated factor 6 (TRAF6) in epileptic patients [23]. The continuous upregulation of inflammatory gene may influence epileptogenesis, so miR 146a may represent a potential tool for therapeutic intervention in epilepsy-related inflammatory responses.

On studying miR 194-5p, it was significantly upregulated in group I compared to control. This disagreed with Wang's study that reported downregulation of miR 194-5p in epileptic patients [27], which could be attributed to the difference in the selection of patients between our study and the mentioned study as they included epileptic patients with different underlying 

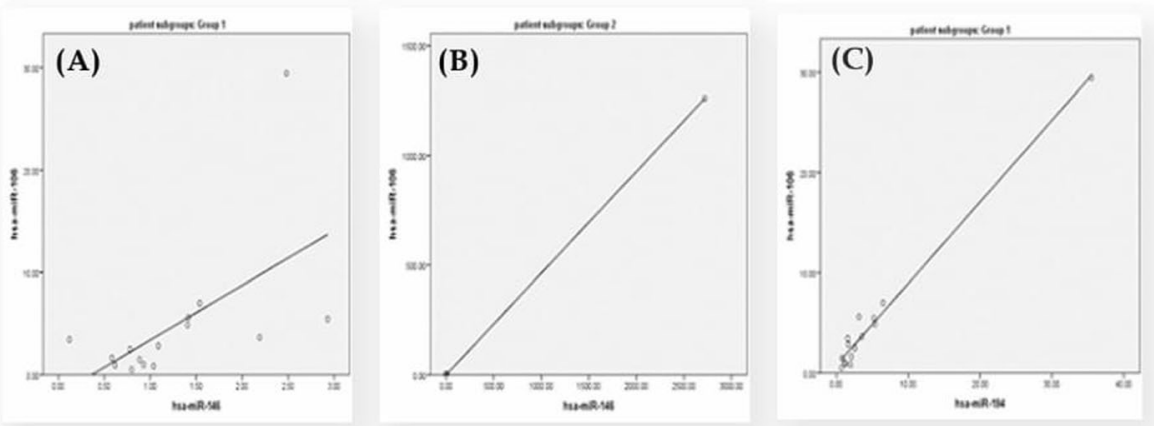

Correlation between $\operatorname{miR} 106 \mathrm{~b}$ and $\mathrm{miR} 146 \mathrm{a}$ in group 1

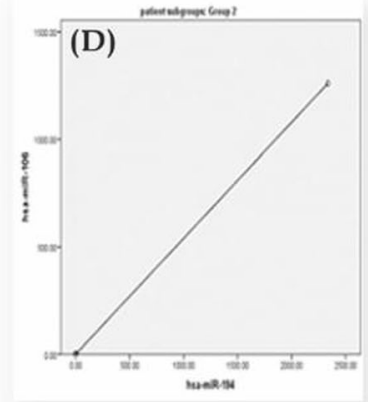

Correlation between miR 106b and miR 194-5p in group 2
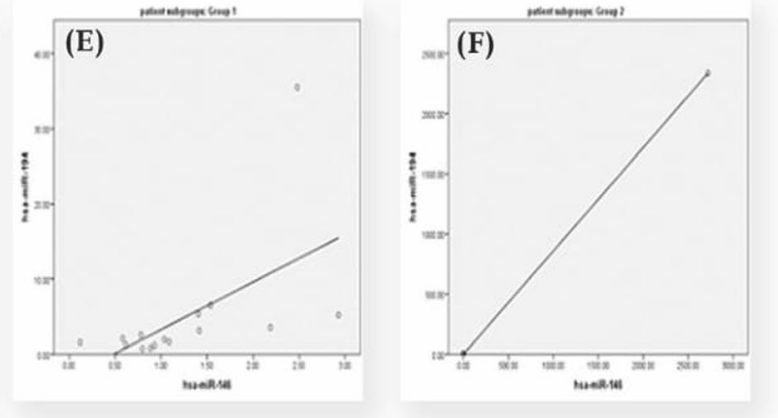

Correlation between

Correlation between miR 106b and miR 146a in group 2

\section{Correlation between miR 106b and miR 194-5p in group 1}

\section{Correlation between miR 194-5p and miR 146a in group 2}

Fig. 2 The correlation between the 3 dysregulated miR (106b, 146a, and 194-5p) with each other in groups I and II

etiologies, while in group I, in our study, only the idiopathic generalized epilepsy was included which is a novel issue in epilepsy research. On the other hand, miR 194-5p was significantly downregulated in group II compared to control.

miR 194-5p has a protective role through inhibition of the mammalian target of rabamycin (mTOR) signaling pathway by targeting SMURF1 (Smad ubiquitin regulatory factor 1) [38]. A consistent anti-epileptogenic role of miR 194-5p could be suggested in epilepsy [39, 40].

Goldberg and Coulter [41] reported that mTOR regulates synaptic plasticity, neuronal structure, and the expression of ion channels and neurotransmitter receptors in the nervous system. Also, LaSarge and Danzer [42] reported that the mTOR pathway may have a vital role in the epileptogenesis process as hyperactivity of the mTOR pathway is associated with abnormal proliferation, differentiation, and cellular alteration contributing to epilepsy.
Our study showed significant downregulation of miR 194-5p in group II compared to group I, which can be attributed to the different target genes for miR 194-5p in generalized and focal epilepsies [43, 44].

In this study, we found a significant negative correlation between the disease duration and the serum level of miR 194-5p in group II; the mean expression of miR 194-5p in group II was significantly downregulated; on the prospect of the predicted protective role of miR 194$5 p$ in epileptogenesis, we can link the bad effect of downregulated miR 194-5p to the long disease duration on the brain and the continuous process of epileptogenesis. Sutula and colleagues reviewed that the longer duration of epilepsy disease is associated with more neuronal loss and more mossy fiber sprouting and granule cell dispersion in the hippocampus. Additionally, longer seizure duration is associated with seizureinduced neurogenesis that occurs in the dentate gyrus 
and loss of the inhibitory interneurons in the hippocampus and dentate gyrus [45].

In our study, we found a significant positive correlation between the three studied miRNAs which was attributed to the predicted common epileptogenic pathways in which the three miRNAs are involved in especially inflammation noticed to be commonly targeted by miR $146 \mathrm{a}$ and $\mathrm{miR} 194-5 \mathrm{p}$. Also, this can indicate the continuous process of epileptogenesis represented by the pro epileptogenic miRNA (miR 106b) which fights against another parallel antiepileptogenic mechanism represented by the antiepileptogenic miRNAs (miR 146a and miR 194-5p). In agreement with our results, Henshall and Meldrum studied gene expression in neuronal loss of temporal lobe epilepsy and found the upregulation of proapoptotic proteins (caspases 2, 3, 6, and 7) [46].

Considering our studied microRNAs as potential biomarkers in epilepsy, miR 106b in the group I was significantly upregulated, with sensitivity $60 \%$ and specificity $66.7 \%$, which suggest the potential use of $\mathrm{miR} 106 \mathrm{~b}$ as a diagnostic biomarker in generalized epilepsy; miR 194$5 \mathrm{p}$ can be also used as a potential diagnostic biomarker in generalized epilepsy with sensitivity $73.3 \%$ and specificity $55.6 \%$.

The small number of patients included in the study (patients per group and patients in the uncontrolled and polytherapy subgroups), in addition to the absence of similar studies focusing on the roles of miR 106b, miR 146a, and miR 194-5p in idiopathic epilepsies, are the most important limitations. However, this study can be considered as a pilot study, which may open the door in the future for studies conducted on large populations to prove the real impact of such miRNAs in epileptogenesis. Also, further studies with assessment of the protective role of miRNAs in epilepsy could be beneficial.

\section{Conclusion}

miR 194-5p and miR 106b may be utilized as potential diagnostic biomarkers in idiopathic generalized epilepsy. $\mathrm{miR} 194-5 \mathrm{p}$ has a protective role in focal epilepsy.

\footnotetext{
Abbreviations

ADNFLE: Autosomal dominant nocturnal frontal lobe epilepsy; AEDs: Antiepileptic drugs; ANOVA: One-way analysis of variance; AUC: Area under the curve; BMI: Body mass index; c2: Chi-square; CDNA: Complementary deoxyribonucleic acid; Cl: Confidence interval: CNS: Central nervous system; COX-2: Cyclooxygenase-2; CUEU: Cairo University Epilepsy Unit; EEG: Electroencephalogram; IL-1: Interleukin-1ß; IL6: Interleukin-6; ILAE: International League Against Epilepsy; IRAK: IL-1 receptor-associated kinases; miR: MicroRNA; miRNA: MicroRNA; mRNA: Messenger RNA; mTOR: Mammalian target of rabamycin; NFB: Nuclear factor kappa-B; OR: Odds ratio; qRT-PCR: Real-time quantitative polymerase chain reaction; RNA: Ribonucleic acid; ROC: Receiver operating characteristic; RT: Reverse transcription; SD: Standard deviation; SMURF1: Smad ubiquitin regulatory factor 1; SPSS: Statistical Package for the Social Science; TAK1: Transforming growth factor beta-activated kinase 1;
}

TGFß: Transforming growth factor-beta; TLR: Toll-like receptor; TRAF6: TNF receptor-associated factor 6

\section{Acknowledgements}

The authors thank the members of the Cairo University Epilepsy Unit (CUEU) for their help in data collection.

\section{Informed consent}

An informed written consent was taken from each patient involved in this study prior to the conduct of any study-related activities. All data obtained from every patient were confidential and were not used outside the study. The patients had the rights to withdraw from the study at any time without giving any reason.

\section{Authors' contributions}

MM contributed to the research idea, data acquisition, data analysis, interpretation, and manuscript reviewing. NTA contributed to the data acquisition, data analysis, and interpretation. DML contributed to the data acquisition, data analysis, interpretation, and manuscript writing and reviewing. NAE and DGA contributed to the interpretation of laboratory data. NM contributed to the data acquisition and data analysis and interpretation. All authors read and approved the final manuscript.

\section{Funding}

The authors have funded the research by themselves.

\section{Availability of data and materials}

The datasets generated and/or analyzed during the current study are not publicly available due to the current Cairo University regulations and Egyptian legislation but they are available by a reasonable request from the corresponding author.

\section{Ethics approval and consent to participate}

All procedures performed in the study were in accordance with the ethical standards of the institutional research committee and with the 1964 Helsinki declaration and its later amendments. Our study was approved by the ethical committee of the Department of Neurology, Faculty of Medicine, Cairo University, in June 2016, but Cairo University does not provide the approval reference number.

\section{Consent for publication}

Not applicable

\section{Competing interests}

None of the authors has any conflict of interest.

\section{Author details}

${ }^{1}$ Neurology Department, Faculty of Medicine, Cairo University, Cairo, Egypt. ${ }^{2}$ Clinical Pathology Department, Faculty of Medicine, Cairo University, Cairo, Egypt.

Received: 20 March 2020 Accepted: 13 July 2020

Published online: 29 July 2020

\section{References}

1. Ngugi AK, Bottomley C, Kleinschmidt I, Sander JW, Newton CR. Estimation of the burden of active and life-time epilepsy: a meta-analytic approach. Epilepsia. 2010;51(5):883.

2. $\quad$ Engel J. Biomarkers in epilepsy: introduction. Biomark Med. 2011;5(5):537.

3. Engel J, Pitkänen A, Loeb JA, Dudek FE, Bertram EH 3rd, Cole AJ, et al. Epilepsy biomarkers. Epilepsia. 2013;54:61.

4. ElSharawy A, Keller A, Flachsbart F, Wendschlag A, Jacobs G, Kefer N, et al. Genome-wide miRNA signatures of human longevity. Aging Cell. 2012;11:607.

5. Henshall DC. MicroRNA and epilepsy. Curr Opin Neurol. 2014;27(2):199.

6. Esteller M. Non-coding RNAs in human disease. Nat Rev Genet. 2011;12:861.

7. Gandhi R, Healy B, Gholipour T, Egorova S, Musallam A, Hussain MS, et al. Circulating microRNAs as biomarkers for disease staging in multiple sclerosis. Ann Neurol. 2013;73:729.

8. Shtilbans A, Henchcliffe C. Biomarkers in Parkinson's disease: an update. Curr Opin Neurol. 2012;25:460. 
9. Tan L, Yu JT, Tan MS, Liu QY, Wang HF, Zhang W, et al. Genome-wide serum microRNA expression profiling identifies serum biomarkers for Alzheimer's disease. JAD. 2014;40(4):1017.

10. Cattani AA, Allene C, Seifert V, Rosenow F, Henshall DC, Freiman TM. Involvement of microRNAs in epileptogenesis. Epilepsia. 2016;57(7):1015.

11. Shao Y, Chen Y. Pathophysiology and clinical utility of non-coding RNAs in epilepsy. Front Mol Neurosci. 2017;10:249.

12. Aronica $\mathrm{E}$, Fluiter $\mathrm{K}$, lyer $\mathrm{A}$, Zurolo E, Vreijling J, van Vliet EA, et al. Expression pattern of miR 146a, an inflammation-associated microRNA, in experimental and human temporal lobe epilepsy. Eur J Neurosci. 2010;31(6):1100.

13. Omran A, Peng J, Zhang C, Xiang QL, Xue J, Gan N, et al. Interleukin-1 $\beta$ and microRNA-146a in an immature rat model and children with mesial temporal lobe epilepsy. Epilepsia. 2012;53(7):1215.

14. Dell'Aversana C, Giorgio C, D'amato L, Lania G, Matarese F, Saeed S, et al. MiR-194-5p/BCLAF1 deregulation in AML tumorigenesis. Leukemia. 2017; 31(11):2315

15. Sonkoly E, Ståhle M, Pivarcsi A. MicroRNAs and immunity: novel players in the regulation of normal immune function and inflammation. Semin Cancer Biol. 2008;18(2):131.

16. Pedersen I, David M. MicroRNAs in the immune response. Cytokine. 2008; 43(3):391.

17. Bhalala OG, Srikanth M, Kessler JA. The emerging roles of microRNAs in CNS injuries. Excellent review covering the role of miRNAs in various neurologic and neurodegenerative disorders as well as prospects for therapy. Nat Rev Neurol. 2013;9:328-39.

18. International League Against Epilepsy (ILAE 1989). Proposal for revised classification of epilepsies and epileptic syndromes. Commission on classification and terminology of the international league against epilepsy. Epilepsia. 1989;30:389.

19. Wang X, Sun L, Zhou Y, Su QJ, Li JL, Ye L, et al. Heroin abuse and/or HIV infection dysregulate plasma Exosomal miRNAs. J Neurolmmune Pharmacol. 2019;10:1007.

20. Liu R, Luo Q, You W, Jin M. MicroRNA-106 attenuates hyperglycemiainduced vascular endothelial cell dysfunction by targeting HMGB1. Gene. 2018;677:142.

21. Garo LP, Murugaiyan G. Contribution of MicroRNAs to autoimmune diseases. Cell Mol Life Sci. 2016;73(10):2041.

22. Raoof R, Bauer $\mathrm{S}$, El Naggar H, Connolly NM, Brennan GP, Brindley E, et al. Dual-center, dual-platform microRNA profiling identifies potential plasma biomarkers of adult temporal lobe epilepsy. EBioMedicine. 2018;38:127.

23. Wang J, Tan L, Tan L, Tian Y, Ma J, Tan CC, et al. Circulating microRNAs are promising novel biomarkers for drug-resistant epilepsy. Sci Rep. 2015;5:10201.

24. Szumilas M. Explaining odds ratios. J Can Acad Child Adolesc Psychiatry. 2010;19(3):227.

25. Mefford HC, Muhle H, Ostertag P, von Spiczak S, Buysse K, Baker C, et al. Genome-wide copy number variation in epilepsy: novel susceptibility loci in idiopathic generalized and focal epilepsies. PLoS Genet. 2010; 20: 6(5): e1000962.

26. Sun J, Cheng W, Liu L, Tao S, Xia Z, Qi L, et al. Identification of serum miRNAs differentially expressed in human epilepsy at seizure onset and post-seizure. Mol Med Rep. 2016;14(6):5318.

27. Wang J, Yu JT, Tan L, Tian Y, Ma J, Tan CC, et al. Genome-wide circulating microRNA expression profiling indicates biomarkers for epilepsy. Sci Rep. 2015;5:9522.

28. Wang H, Liu J, Zong Y, Xu Y, Deng W, Zhu H, et al. Brain Res. 2010;1357:166.

29. Tian $Q$, Xiao Q, Yu W, Gu M, Zhao N, Lü Y. The inhibition of transforming growth factor beta-activated kinase 1 contributed to neuroprotection via inflammatory reaction in pilocarpine-induced rats with epilepsy. Neurosci. 2016:325:111.

30. Dobolyi A, Vincze C, Pál G, Lovas G. The neuroprotective functions of transforming growth factor beta proteins. Int J Mol Sci. 2012;13(7):8219.

31. Henshall DC, Simon RP. Epilepsy and apoptosis pathways. J Cereb Blood Flow Metab. 2005;25(12):1557.

32. Huang W, Li Z, Zhao L, Zhao W. Simvastatin ameliorate memory deficits and inflammation in clinical and mouse model of Alzheimer's disease via modulating the expression of miR 106b. Biomed Pharmacother. 2017;92:46.

33. Narkilahti S, Pitkänen A. Caspase 6 expression in the rat hippocampus during epileptogenesis and epilepsy. Neuroscience. 2005;131(4):887.

34. Brett JO, Renault VM, Rafalski VA, Webb AE, Brunet A. The microRNA cluster miR 106b 25 regulates adult neural stem/progenitor cell proliferation and neuronal differentiation. Aging (Albany NY). 2011;3(2):108.
35. Otaegui D, Baranzini SE, Arman R, Calvo B, Munoz-Culla M, Khankhanian P, et al. Differential micro RNA expression in PBMC from multiple sclerosis patients. PLoS One. 2009:4:e6309.

36. Sheedy FJ, O'Neill LA. Adding fuel to fire: microRNAs as a new class of mediators of inflammation. Ann Rheum Dis. 2008;67(Suppl. 3):iii50.

37. Taganov KD, Boldin MP, Chang KJ, Baltimore D. NF-kappa B-dependent induction of microRNA miR 146, an inhibitor targeted to signaling proteins of innate immune responses. Proc Natl Acad Sci U S A. 2006;103:12481.

38. Chong ZZ, Shang YC, Zhang L, Wang S, Maiese K. Mammalian target of rapamycin: hitting the bull's-eye for neurological disorders. Oxidative Med Cell Longev. 2010;3:374.

39. McDaniel SS, Wong M. Therapeutic role of mammalian target of rapamycin (mTOR) inhibition in preventing epileptogenesis. Neurosci Lett. 2011;497:231.

40. Russo E, Citraro R, Constanti A, De Sarro G. The mTOR signaling pathway in the brain: focus on epilepsy and epileptogenesis. Mol Neurobiol. 2012;46(3): 662.

41. Goldberg EM, Coulter DA. Mechanisms of epileptogenesis: a convergence on neural circuit dysfunction. Nat Rev Neurosci. 2013;14(5):337.

42. LaSarge CL, Danzer SC. Mechanisms regulating neuronal excitability and seizure development following mTOR pathway hyperactivation. Front Mol Neurosci. 2014;7:18,

43. Panjwani N, Wilson MD, Addis L, Crosbie J, Wirrell E, Auvin S, et al. A microRNA-328 binding site in PAX6 is associated with centrotemporal spikes of rolandic epilepsy. Ann Clin Transl Neurol. 2016;3(7):512.

44. Strehlow V, Swinkels ME, Thomas RH, Rapps N, Syrbe S, Dorn T, et al. Generalized epilepsy and myoclonic seizures in 22q11.2 deletion syndrome. Mol Syndromol. 2016;7(4):239.

45. Sutula TP, Hagen J, Pitkänen A. Do epileptic seizures damage the brain? Curr Opin Neurol. 2003;16(2):189.

46. Henshall DC, Meldrum BS. Cell death and survival mechanisms after single and repeated brief seizures. In: Noebels JL, Avoli M, Rogawski MA, Olsen RW, Delgado-Escueta AV, editors. Jasper's basic mechanisms of the epilepsies.4th ed. USA: Oxford University Press; 2012. p. 262-76.

\section{Publisher's Note}

Springer Nature remains neutral with regard to jurisdictional claims in published maps and institutional affiliations.

\section{Submit your manuscript to a SpringerOpen ${ }^{\circ}$ journal and benefit from:}

- Convenient online submission

Rigorous peer review

- Open access: articles freely available online

- High visibility within the field

- Retaining the copyright to your article

Submit your next manuscript at $\boldsymbol{\nabla}$ springeropen.com 